\title{
La formación inicial de profesores en las Escuelas Normales
}

Mireya Chapa Chapa

Escuela Normal Pablo Livas

Manuel Flores Fahara

Escuela de Educación, Humanidades y Ciencias Sociales

Instituto Tecnológico y de Estudios Superiores de Monterrey

\section{Resumen}

2

1 tema de la formación inicial de los profesores de educación básica en México y en el plano internacional, es eje del debate educativo actual. El artículo incluye avances de un estudio acerca del desarrollo de competencias profesionales en el período inicial de formación, mediante un seguimiento a las actividades relacionadas con la práctica profesional en los ocho semestres de la escuela normal. El problema de investigación tiene como pregunta central ¿De qué manera se desarrollan las competencias profesionales en la formación inicial docente en la escuela normal?. Para la revisión de la literatura, se exploró el panorama internacional y nacional en la formación de profesores, la teoría sobre la formación en competencias, el análisis de la orientación del Plan de estudios 2012 para la formación de maestros de educación básica y el papel del trayecto formativo de práctica profesional. El estudio considera un enfoque cualitativo de caso único. Se presentan algunos resultados preliminares obtenidos a partir del análisis de documentos elaborados por los estudiantes en el $1^{\circ} \mathrm{y}$ $2^{\circ}$ semestre de la formación inicial docente: análisis de caso a partir de una historia docente y registros narrativos de las visitas de observación a tres escuelas primarias.

Palabras clave: formación de profesores, educación normalista, competencias profesionales. 


\section{Introducción}

La transformación constante de la sociedad exige que el paradigma de formación de los profesores se sustente en presupuestos teórico-prácticos complejos y completos, pues solamente de esa forma será posible responder a las múltiples y crecientes demandas de la educación, la competencia, profesionalidad y la capacidad de entrega que se le exige al profesorado, que hace recaer sobre sus espaldas (y la de los encargados del proceso de formación) una gran responsabilidad (Delors, 1996). La formación de profesores representa la principal esperanza para aquellos que confiamos en las capacidades de los propios docentes para hacer propuestas de mejora educativa (Marcelo, 2002).

La problemática de la formación del profesorado requiere reflexionar sobre el conocimiento de las competencias profesionales que se exigen y son necesarias para el desarrollo de su trabajo y el conocimiento profesional necesario para desempeñar con calidad la actividad docente (Pires, 2012).

Existe evidencia para considerar la formación inicial docente como un proceso clave en el desarrollo educativo (García, 1995; Pires, 2012; Ferry, 1987; De la Torre y Barrios, 2002; Imbernón, 2001) además de que los resultados de análisis de los procesos de formación de profesores (Marcelo, 2002; Pires, 2012; Ruffinelli, 2014) y de evaluaciones estandarizadas indican problemáticas latentes (SEP, 2014; OECD, 2014). A la vez, existe información que respalda los procesos de seguimiento, evaluación y retroalimentación del desarrollo de competencias profesionales como un factor que impacta en el trabajo del profesor y los resultados de sus estudiantes (Manzi, 2011).

A partir de estos antecedentes, se plantea la siguiente pregunta de investigación: ¿ $\mathrm{De}$ qué manera se desarrollan las competencias profesionales en la formación inicial docente en la escuela normal? (Figura 1).

De la pregunta de investigación se derivan dos preguntas secundarias: (1) ¿Cuáles son las competencias profesionales que se desarrollan durante la formación inicial docente en la escuela normal? y (2) ¿Cuáles son los factores que intervienen en el desarrollo de competencias profesionales durante la formación inicial docente en la escuela normal?

El propósito del estudio es: conocer, a partir del seguimiento al proceso formativo de un grupo de estudiantes de la generación 2012-2016, cómo se desarrollan las competencias profesionales durante la formación inicial docente en una escuela normal. Se espera describir el proceso de formación, desde la perspectiva de los docentes en formación y los profesores involucrados, así como las evidencias de aprendizaje que van dando cuenta del nivel de logro de las competencias profesionales. Además, se busca identificar cuáles son los factores que intervienen en el desarrollo de competencias profesionales y las diferentes estrategias aplicadas para realizar el seguimiento y valoración del progreso de éstas. Con los resultados de la investigación, se pretende aportar mayor evidencia empírica sobre el tema de formación inicial docente en las escuelas normales, el desarrollo de competencias profesionales y contribuir a la generación de conocimiento a partir de la apli- 
REVISTA DE INVESTIGACIÓN EDUCATIVA DE LA REDIECH N. 10

ISSN: 2007-4336

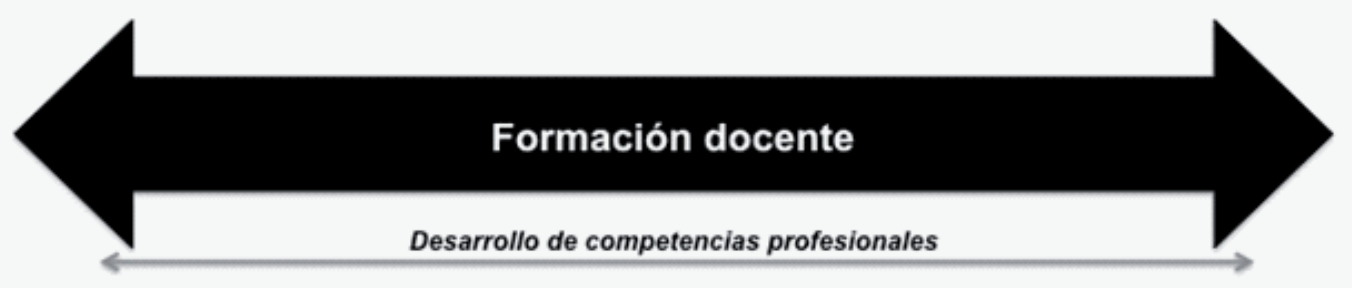

Iniciación a la enserianza

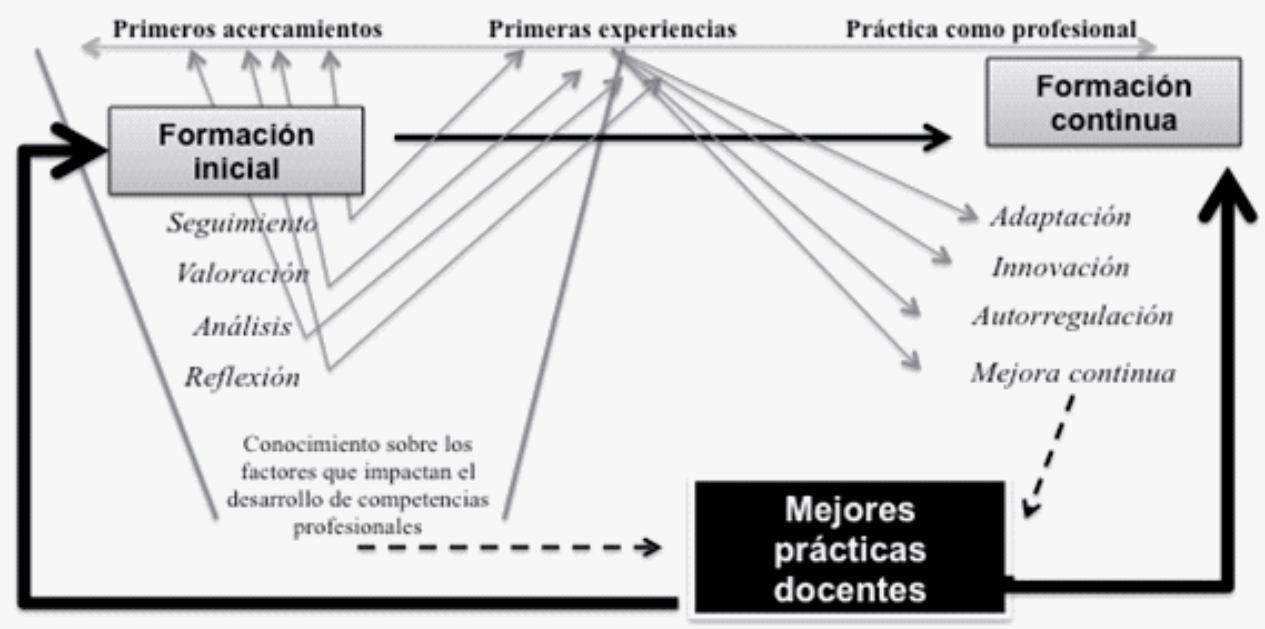

Figura 1. Aspectos para definir el problema de investigación. Elaboración propia.

cación del Plan de estudios 2012 para la formación de maestros de educación primaria en México, del cual existe escasa literatura.

En general, es variada la información sobre la formación de profesores a nivel nacional e internacional. Desde hace una década se habla del malestar que se vive en las escuelas normales, en los procesos de trabajo, en los referentes de valores, la pérdida de pertenencia, de valía, status y reconocimiento político y social. Jiménez (2003) lo define como malestar identitario en la educación normal. Sandoval (1993) analiza cómo el trabajo del maestro es objeto de una desvalorización social que, incluso ha repercutido en la autoestima de los propios docentes. Asimismo señala que si no se considera de manera integral la formación de maestros y su futuro espacio de trabajo y no se revalora efectivamente la función del maestro en lo profesional y en lo económico, los problemas seguirán. De Ibarrola (1995) también aborda la problemática situación de la formación de maestros y la caracteriza por un intrincado conjunto de procesos y programas institucionales que lejos de facilitar una reforma integral, lo que hacen es obstaculizarla.

Paz, Venet, Márquez y Orozco (2011) refieren que aunque la investigación educativa ha favorecido la comprensión de los procesos de formación de profesores en fechas recientes, se siguen presentando insuficiencias en las prácticas pedagógicas, en la formación inicial, continua y permanente de los docentes. 
Greybeck, Moreno y Peredo (1998) entienden la formación inicial como la preparación profesional para la docencia en algún nivel $o$ área educativa, la cual se formaliza a través de la obtención de un título de licenciatura. En México esta formación profesional, realizada históricamente en las escuelas normales, ha pasado por diferentes etapas, desde el "cómo hacer" con planes de estudios a partir de la técnica de la enseñanza hasta al decreto del Acuerdo 649, por el que se establece el Plan de estudios para la formación de maestros de educación primaria 2012, que busca el desarrollo de competencias genéricas y profesionales para la docencia.

Los retos que la formación inicial docente enfrenta incluyen la formulación de los programas según las concepciones asumidas, la búsqueda de estrategias conducentes a alcanzar los propósitos formativos y la previsión de acciones para la formación de formadores (Greybeck, Moreno y Peredo, 1998).

La sabiduría que otorga la práctica, es de acuerdo a Shulman (2001) la más rica fuente del conocimiento de un docente, pero al mismo tiempo es la menos codificada de todas. Al documentar la manera en que a través de la preparación de las jornadas de observación y práctica docente, los docentes formadores propician el desarrollo de las habilidades pedagógicas, será posible establecer una metodología de enseñanza. Además el propiciar que los estudiantes sistematicen sus experiencias y las analicen a través de la reflexión sobre su práctica, favorecerá su desarrollo como profesionales de la educación.
Aprender haciendo, en este caso el aprender a enseñar enseñando, requiere del perfeccionamiento de los métodos y formas de organización de la enseñanza y del trabajo colectivo en las escuelas normales. Perrenoud (2001) explica que en la formación inicial docente es necesaria una trasposición didáctica fundada en el análisis de las prácticas y sus transformaciones, lo que requiere desarrollar en los estudiantes la habilidad de realizar una transferencia didáctica de las prácticas efectivas de un gran número de profesores, respetando la diversidad de condiciones en las que se trabaja.

Entender el desarrollo de las competencias docentes es una tarea compleja. En la formación de los profesores influyen diferentes elementos. Fierro, Fortoul y Rosas (2008) mencionan que para entender el trabajo de un profesor es necesario considerar seis dimensiones: personal, valoral, didáctica, institucional, interpersonal y social. Estas dimensiones se ponen en juego en la relación pedagógica, que es ese momento en el que el docente trabaja, interactúa y aprende con sus alumnos. Esta tarea no es fácil, porque esas prácticas se adquieren no solo de los profesores de educación primaria a los que se observa en las actividades de acercamiento a la práctica, sino (y en mayor medida) de los mismos docentes formadores de la escuela normal, que basados en su propia experiencia como ex profesores de educación básica u observadores, pueden proporcionar una visión prescriptiva del oficio, con lo que se corre el riesgo de la reproducción.

Para Perrenoud (2001) describir las condiciones y las dificultades del trabajo real de 
REVISTA DE INVESTIGACIÓN EDUCATIVA DE LA REDIECH N. 10

los docentes es la base de la innovación y el primer paso a la mejora de las prácticas.

\section{Apunte metodológico}

El estudio tiene un enfoque cualitativo, como estudio de caso único y se realiza en la Escuela Normal Profesores Líderes. Esta institución formadora de docentes es de sostenimiento público de tipo estatal. Se ubica en un municipio del norte de un estado del noreste de México y atiende a 350 estudiantes en la Licenciatura en Educación Primaria. Se selecciona por la apertura de sus integrantes a participar y su pertinencia para el estudio (Stake, 2007). El desarrollo de las actividades del estudio considera la recopilación de información de agosto de 2012 a julio de 2016, período de tiempo regular en el que una cohorte generacional realiza la licenciatura en educación primaria. En este artículo se reportan los resultados obtenidos a febrero de 2015.

Se consideró la muestra por conveniencia (Hernández, Fernández y Baptista, 2010) para la elección de participantes, ya que son los casos disponibles a los que se tiene acceso. Los participantes son los estudiantes que conforman el grupo $\mathrm{B}$ de la licenciatura en educación primaria en el transcurso de su trayecto formativo. En el período escolar febrero-julio 2015, son 27 estudiantes, 12 hombres y 15 mujeres que ingresaron a la ENPL en agosto de 2012 y han cursado la carrera de forma regular. Sus edades fluctúan entre los 20 y 42 años de edad. Otra de las características de los participantes es que corresponden a lo que se denomina muestra diversa o de máxima variación, ya que representan distintas perspectivas y características de los estudiantes normalistas, lo que representa la complejidad del fenómeno estudiado.

Al partir de la premisa de describir cómo se desarrollan las competencias profesionales durante la formación inicial docente en la escuela normal considerando el propio proceso de formación de los estudiantes, se busca generar teoría basada en las experiencias, por lo que se propone el diseño de teoría fundamentada en la realización del estudio. La teoría fundamentada utiliza un procedimiento sistemático cualitativo para generar una teoría que explique en un nivel conceptual una acción, interacción o tema específico (Glasser y Strauss, 1967).

La entrevista, la observación participante, la revisión de documentos y el grupo focal son las estrategias para la recolección de datos a lo largo de la formación inicial de los estudiantes normalistas (Tabla 1).

\section{Avances en los resultados de la investigación}

De acuerdo a lo planteado en la metodología de este estudio, se presentan resultados preliminares a partir del primer análisis de las producciones de los estudiantes elaboradas en los primeros semestres de la formación inicial. Como primer paso para su realización, se identificaron las competencias genéricas y profesionales que plantea el Plan de estudios 2012 para la formación de maestros de educación primaria, así como las unidades de competencia que considera cada una de éstas y se organizaron en una tabla de doble entrada. Cabe destacar que en este estudio se considera solamente el análisis del desarrollo de las competencias profesionales. 
Tabla 1. Instrumentos para la recolección de datos

\begin{tabular}{|c|c|c|c|c|}
\hline Técnica: & $\begin{array}{l}\text { Observación } \\
\text { participante }\end{array}$ & $\begin{array}{l}\text { Análisis de } \\
\text { documentos }\end{array}$ & Entrevista & Grupo focal \\
\hline Proceso: & Actuación & Evaluación & Regulación & Desarrollo \\
\hline Aspecto: & Puesta en práctica & $\begin{array}{l}\text { Evidencias de } \\
\text { desarrollo }\end{array}$ & $\begin{array}{c}\text { Valoración del } \\
\text { desarrollo }\end{array}$ & $\begin{array}{l}\text { Actividades de } \\
\text { formación }\end{array}$ \\
\hline Propósito: & $\begin{array}{c}\text { Reconocer la } \\
\text { puesta en práctica } \\
\text { de competencias } \\
\text { profesionales en la } \\
\text { actuación en una } \\
\text { situación real. }\end{array}$ & $\begin{array}{c}\text { Identificar las } \\
\text { evidencias que dan } \\
\text { cuenta del } \\
\text { desarrollo de } \\
\text { competencias } \\
\text { profesionales } \\
\text { durante la } \\
\text { formación inicial. }\end{array}$ & $\begin{array}{l}\text { Indagar acerca de } \\
\text { la valoración que } \\
\text { estudiantes y } \\
\text { profesores realizan } \\
\text { del proceso de } \\
\text { desarrollo de } \\
\text { competencias. }\end{array}$ & $\begin{array}{l}\text { Identificar las } \\
\text { actividades de } \\
\text { formación que } \\
\text { favorecen el } \\
\text { desarrollo de } \\
\text { competencias } \\
\text { profesionales. }\end{array}$ \\
\hline \multirow{4}{*}{ Fuentes } & \multirow{4}{*}{$\begin{array}{c}\text { Bitácora de campo } \\
\text { Notas } \\
\text { Videos } \\
\text { Fotografias }\end{array}$} & Registro narrativo & Estudiantes & \multirow{4}{*}{$\begin{array}{c}\text { Estudiantes } \\
\text { Profesores de la } \\
\text { escuela normal }\end{array}$} \\
\hline & & Registro & $\begin{array}{l}\text { Profesores de } \\
\text { educación }\end{array}$ & \\
\hline & & fotográfico & primaria & \\
\hline & & $\begin{array}{l}\text { Fichas de } \\
\text { evaluación }\end{array}$ & $\begin{array}{l}\text { Profesores de la } \\
\text { escuela normal }\end{array}$ & \\
\hline
\end{tabular}

Fuente: Elaboración propia.

Como segundo paso se identificaron en los programas de estudio, las competencias profesionales que se espera desarrollar con los cursos: Observación y análisis de la práctica educativa y Observación y análisis de la práctica escolar ( $1^{\circ}$ y $2^{\circ}$ semestre). Además, se concentraron las competencias esperadas de cada curso, con el propósito de identificar su relación. Se registraron también los productos esperados con el propósito de destacar su relación con actividades a realizar en el contexto de las escuelas primarias y se seleccionaron aquellos que directamente se derivaron de éstas.

Una vez identificadas las competencias y productos que evidencian su desarrollo se procedió al análisis de estos. Cabe destacar que en el trayecto de Práctica profesio- nal y en los cursos del primer año de formación, la competencia profesional que se busca desarrollar es la misma. En el primer semestre como competencia del curso: $O b$ serva y analiza con rigurosidad las diferentes dimensiones sociales que se articulan con la educación, la comunidad, la escuela y los sujetos que confluyen en ella. En el segundo semestre se trabaja con la competencia del curso: Profundiza acerca de las relaciones entre la escuela y la comunidad, la gestión y organización institucional, así como en las interacciones pedagógicas que se desarrollan al interior del aula de clase.

El análisis curricular permitió observar que el desarrollo de esta competencia es paulatino y tiene relación con los conocimientos teóricos y metodológicos aborda- 
dos en los dos primeros cursos del trayecto formativo. A partir del análisis de documentos, se detectó que los principales aprendizajes reportados por los estudiantes son que la práctica docente es una tarea compleja que se ve influida positiva o negativamente por el contexto y que no hay situaciones ideales. Además, los jóvenes registraron que aprendieron que aunque en el salón de clases solamente estén los niños y el profesor, lo que viven los alumnos en casa se refleja en el salón, en el comportamiento, lenguaje $\mathrm{y}$ actitudes de los niños.

Otro aspecto relevante, mencionado por el $85 \%$ de los estudiantes es que la profesión docente no es tarea fácil, sino que está llena de retos que incluyen el mantenerse actualizado, conocer a los alumnos y sus padres, tener una buena relación con otros profesores y adaptarse al contexto escolar, pero utilizando todos los recursos disponibles para cumplir con los propósitos educativos. Se destaca también que más que solamente caracterizar a un profesor como bueno o malo, analizan desde las diferentes dimensiones su situación, concluyendo que la actuación de un profesor depende de diferentes factores.

De manera específica sobre la práctica docente, la mayor parte de los estudiantes concuerda en que además de ser compleja, está en constante transformación por depender de las condiciones y circunstancias que rodean al docente y sus alumnos.

\section{Conclusiones}

Los hallazgos preliminares indican que, a partir de lo observado en la escuela primaria, los estudiantes hacen una relación entre los conceptos teóricos revisados en la escuela normal y la realidad, pero lo más significativo para ellos parte de la práctica cotidiana de los profesores. Se detectó también que la incorporación de las dimensiones de análisis de la práctica docente propuestas por Fierro, Fortoul y Rosas (2008) funcionan como una especie de filtro, a través del cual los estudiantes consideran diferentes factores y analizan lo que acontece en la escuela, siendo capaces de retomar aspectos positivos para su formación y rechazar comportamientos que consideran poco significativos.

A partir del trabajo realizado, es posible apreciar que el enfoque con el que está diseñado el trayecto formativo de Prácticas profesionales transforma la manera en la que el profesor en formación va conformando su propia práctica docente. El espacio otorgado por el plan de estudios 2012 para que el estudiante normalista analice la práctica educativa de las instituciones en las que ha sido estudiante y la revisión de metodología le proporcionan conocimientos y competencias que le permiten analizar la realidad desde una perspectiva distinta. El impacto de la primera jornada de observación en el pensamiento de los estudiantes sobre la práctica docente es importante, ya que les permite analizar la realidad cotidiana de las escuelas, pero la metodología con la que se lleva a cabo permite que en lugar de ser una confrontación con un ideal, sea un espacio para reflexionar sobre los retos de la profesión y las fortalezas y áreas de oportunidad que los estudiantes poseen, además de ser el punto de partida para iniciar con la construcción del tipo de profesor que desean ser, generándose transformaciones en las 
dimensiones personal y valoral de su práctica docente.

Estos resultados son preliminares y requieren de una delimitación más concreta y relacionada de manera particular con las competencias profesionales que se espera los estudiantes alcancen al término de este período de formación.

\section{Bibliografía}

De Ibarrola, M. (1995) Formación de docentes. Reforma educativa y crisis económica en México en el marco del TLC en SEP - UPN, Formación docente, modernización educativa y globalización, México, pp. $73-86$.

De la Torre, S. y Barrios, O. (2002) Curso de formación para educadores. Brasil: Madras Ltd.

Delors, J. (1996) La educación encierra un tesoro Informe a la UNESCO de la Comisión Internacional sobre la educación para el Siglo XXI. España: Santillana UNESCO.

Ferry, G. (1987) Pedagogía de la formación. Argentina: Novedades Educativas.

Fierro, C., Fortoul, B. y Rosas, L. (2008) Transformando la práctica docente Una propuesta basada en la investigación-acción. (1 ${ }^{\text {a }}$ reimpresión). México: Paidós.

García, C. (1995) Formación de profesores para la reforma educativa. Brasil: Porto Editora.

Glaser, B. y Strauss, A. (1967) The discovery of grounded theory: strategies for qualitative research. Estados Unidos: Aldine Publishing Company

Greybeck, D., Moreno, M. y Peredo, M. (1998) Reflexiones acerca de la formación de docentes, Educar, (5)15 -22 .

Hernández, R. Fernández, C. y Baptista, P. (2010) Metodología de la investigación ( $5^{\mathrm{a}}$ ed.). México: McGrawHill.

Imbernón, F. (2001) Formación docente y profesión: formar para la reforma y la incertidumbre. Brasil: Cortez.
Jiménez, M. (2003) Identidad de los profesores de educación básica y normal en Sujetos, actos y procesos de formación II, México: Consejo Mexicano de Investigación Educativa. pp. 609 - 617.

Manzi, J. (coord.). (2011) ¿Qué características de la formación inicial de los docentes se asocian a mayores avances en su aprendizaje de conocimientos disciplinarios? Informe final. Chile: Ministerio de Educación.

Marcelo, C. (2002) La formación inicial y permanente de los educadores. En Consejo Escolar del Estado. Los educadores en la sociedad del siglo XXI. España: Ministerio de Educación, Cultura y Deporte.pp. 161 - 194.

Organization for the Economic Cooperation and Development [OECD]. (2014). New Insights from TALIS 2013: Teaching and Learning in Primary and Upper Secondary Education. OECD Publishing. http:// dx.doi.org/10.1787/9789264226319-en

Paz, I., Venet, R., Ramos, G., Márquez, A. (2011) Formando al educador del siglo XXI. Reflexiones, experiencias y propuestas pedagógicas. Cuba: Ministerio de educación.

Perrenoud, P. (2001) La formación de los docentes en el siglo XXI. Revista de Tecnología Educativa (3), 503 $-523$.

Pires, L. (2012) La formación inicial de los profesores y las profesoras. Rizoma freireano (12), 1-18.

Ruffinelli, A. (2014) ¿Qué aprenden los docentes en su primer año de ejercicio profesional?: representaciones de los propios docentes principiantes. Pensamiento Educativo. Revista de Investigación Educacional Latinoamericana, 51 (2), 56-74.

Sandoval, E. (1993) Identidad y memoria del normalismo en Fundación SNTE II. México: SNTE. pp. 226 - 236.

Secretaría de Educación Pública [SEP]. (2014) Total y porcentajes de sustentantes por grupo de desempeño $y$ entidad federativa. Recuperado el 23 de marzo de 2015 en http://servicioprofesionaldocente.sep.gob. $\mathrm{mx} / \mathrm{ba}$ /estadisticas_concurso_a/

Shulman, L. (2001) Conocimiento y enseñanza. Estudios públicos (83), 163 - 195.

Stake, R. (2007). (4a ed.) Investigación con estudio de casos. España: Morata. 